\title{
Trabajos Originales \\ Caracterización de casos de Maltrato Infantil, Dirección de Medicina Forense de Tegucigalpa
}

\section{Characterization of cases of Child Maltreatment, Dirección de Medicina Forense of Tegucigalpa.}

Dra. Norma Dilenia Castillo Godoy ${ }^{1}$, Dra. Mireya Matamoros Zelaya ${ }^{2}$, Dr. Gustavo Roque Pacheco ${ }^{3}$, Dra. Semma Julissa Villanueva ${ }^{4}$.

\begin{abstract}
${ }^{1}$ Residente de Posgrado Medicina Legal y Forense, Universidad Nacional Autónoma de Honduras y Dirección de Medicina Forense; ${ }^{2}$ Master en Microbiología y Antropología y Genética Forense, Dirección de Medicina Forense, Tegucigalpa. ${ }^{3}$ Médico Forense, Departamento de Clínica Forense, Dirección de Medicina Forense, Tegucigalpa, ${ }^{4}$ Especialista en Anatomía Patológica, Dirección de Medicina Forense, Tegucigalpa. y Posgrado en Medicina Legal y Forense, Universidad Nacional Autónoma de Honduras.
\end{abstract}

*Correspondencia a Norma Castillo Godoy: dileniagodoy@gmail.com

\section{REFERENCIA}

Castillo-Godoy ND, Matamoros M, RoquePacheco G, Villanueva SM. Caracterización de casos de maltrato infantil, Dirección de Medicina Forense de Tegucigalpa. Rev. cienc. forenses Honduras. 2018; 4(2):2-10.

Los autores declaran que no tienen conflicto de intereses en la publicación de este artículo.

Agradecimientos al Licenciado Asael Alonzo Matamoros, por su apoyo en el análisis estadístico.

RECIBIDO: Noviembre 2017

ACEPTADO: Agosto 2018

\section{RESUMEN:}

El maltrato infantil es un importante problema de salud pública que afecta la salud y vida de las víctimas, que está presente en todas las culturas y estratos socio económicos y que tiene graves consecuencias de salud para las víctimas. Este estudio caracterizó al niño maltratado evaluado en la Clínica Forense de
Tegucigalpa, el período 2010 al 2015. Mediante un estudio descriptivo, retrospectivo; se analizaron 267 casos de maltrato infantil. El 95.5\% presentó maltrato físico por transgresión. Las características relevantes de las víctimas de maltrato infantil fueron: En su mayoría niñas (57,3\%) $(t=2,408$, $d f=266$, pvalor $=0,016$ ), escolares, con 8.6 años de edad promedio, el $46 \%$ convivían en el seno de su familia nuclear, el 84,3\% fueron agredidas en su vivienda, la madre fue la principal agresora. El maltrato físico por transgresión fue el más reportado, y el cinturón el principal objeto de agresión.

\section{PALABRAS CLAVE}

Maltrato infantil, Maltrato por transgresión, Maltrato por supresión, Maltrato por omisión.

\section{ABSTRACT}

Child maltreatment is an important public health problem that affects the health and lives 
of victims, it is present in all cultures and socioeconomic strata and has serious health consequences for victims

This study characterized the abused child evaluated in the in the Department of Forensic Clinic of Tegucigalpa in the period 2010 to 2015. 95.5\% presented physical abuse by transgression, the belt was the main object of aggression. The relevant characteristics of the victims of child abuse were: Mostly girls ( $t=$ 2.408, $d f=266$, pvalor $=0.016)$, schoolchildren, with 8.6 years of age on average, $46 \%$ lived in their nuclear family, $84.3 \%$ were attacked in their home, the mother was the main aggressor. Physical abuse by transgression was the most reported.

\section{KEYWORDS}

Child abuse, Abuse by transgression, Abuse by suppression, Abuse by omission.

\section{INTRODUCCIÓN}

El maltrato infantil, está presente en todas las culturas y estratos socio económicos y tiene graves consecuencias de salud para las víctimas ${ }^{1}$. En el área médico legal las evaluaciones por maltrato infantil se realizan de manera rutinaria, sin embargo, en nuestro país se conoce muy poco acerca de las características sociodemográficas, tipos de maltrato y lesiones que presentan las víctimas objeto de evaluación médico legal.

El Código de la Niñez y Adolescencia de Honduras establece que "el maltrato infantil es toda acción u omisión que violenta los derechos y el bienestar de los niños, afectando su salud física, mental, así como su estado emocional, intelectual y psicosocial", la ley hondureña, además estipula la obligatoriedad de la notificación ${ }^{2}$, sin embargo la mayoría de los casos no se reportan a las autoridades, o se disfrazan como accidentes domésticos y de esta forma pasan desapercibidos ${ }^{3}$, lo que dificulta conocer la magnitud real de este problema.

Este trabajo describe el tipo de maltrato, así como las características de los niños maltratados y sus lesiones, evaluados en la Clínica Forense de Tegucigalpa entre el 2010 y 2015.

\section{MÉTODOLOGÍA}

Se realizó un estudio descriptivo, retrospectivo, con la revisión de 267 expedientes médico legales de casos de menores evaluados por maltrato infantil en el Departamento de Clínica Forense de Tegucigalpa, en el período comprendido del 01 de enero de 2010 al 31 de diciembre de 2015. Se excluyeron del estudio los casos de delitos sexuales. La información se recopiló en Microsoft Excel $2016^{\circledR}$, se analizaron parámetros de estadística descriptiva utilizando el programa R Core Team $2018{ }^{4}$. Las variables estudiadas fueron: edad, sexo, grupo etario, procedencia, tipo de familia en la que convivía, antecedentes de agresiones, evaluaciones médicas previas a la evaluación médico legal, tiempo transcurrido entre el hecho y la evaluación médico legal, lugar donde ocurrió la agresión, tipo de maltrato sufrido, tipo de lesiones y su ubicación anatómica, instrumento de lesión y agresor.

\section{RESULTADOS}

El $57.3 \%$ (153) de los casos fueron femeninos y el 42.7\% (114) masculinos. La edad promedio fue de 8.6 años en las niñas y de 7.8 años en los niños. Respecto a la distribución por grupo etario, los escolares presentaron el $45 \%$ de los casos. La distribución por grupo etario y sexo, se muestra en la Figura 1

El 86\% (230) procedían del área urbana del país y 


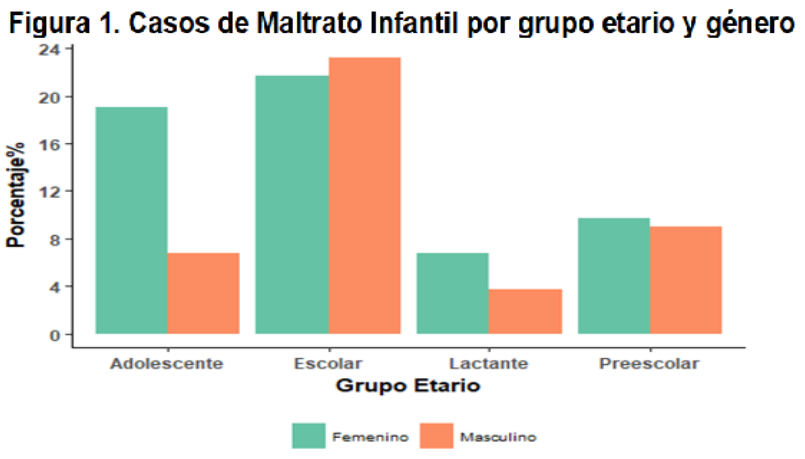

convivían con su familia nuclear (46\%), el 25.8\% (69 casos) con su familia extendida y el $13,5 \%$ convivían en familias desintegradas. El 60\% (160) de los niños afirmaron haber sido agredidos anteriormente, en el $21 \%$ (34) de ellos se encontraron cicatrices antiguas. El 6\%(16) recibieron atención médica previa a la evaluación médico legal, de los cuales el $87,5 \%$ recibieron la atención en una institución pública de salud, el $37,5 \%$ requirieron procedimientos quirúrgicos menores y $18,75 \%$ requirieron hospitalización. El tiempo transcurrido entre el hecho y la atención médica fue menor de 24 horas, en el $75 \%$ de los casos.

En el $84.3 \%$ (225) la agresión ocurrió en la vivienda de la víctima, el 71,5\% fueron referidos a evaluación médico legal por la Fiscalía Especial de la Niñez; el tiempo promedio transcurrido entre el hecho y la evaluación médico legal fue de 19 días; el 50\% (134) de los casos fueron evaluados en un periodo menor de tres días.

La distribución de los tipos de maltrato infantil se muestra en el Cuadro 1. Se observó que la transgresión corresponde al $41.9 \%$ de los casos, la omisión al $7.5 \%$ y la supresión al $0.7 \%$; el $28.5 \%$ se presentaron asociados a transgresión y supresión.

En los casos con maltrato infantil por omisión, se observó que el $27 \%$ (15) había sido abandonado, el $25 \%$ (14) no iba a la escuela y en tres casos se reportó más de una forma de omisión. Cuadro 2.

En los casos que presentaron maltrato infantil por supresión, el $74.5 \%$ (76) correspondió a medidas correctivas o disciplinarias inadecuadas para la edad. Cuadro 3.

En el maltrato infantil por transgresión, el 95.5\% (255) presentó maltrato físico, y en la mitad de estos, se observó maltrato físico crónico, respecto al cuerpo, objeto o mecanismo de lesión, relatado por el menor o su acompañante, el cinturón fue utilizado en el $40 \%$ (107) de los casos, el golpe con lazo en el $12.5 \%$ (34) y el golpe con palo en $4.5 \%$ (12) de los casos.

El 9,7\% (26) reportó malos tratos verbales, y de estos, el $46.1 \%$ (12) fue por ofensas, humillaciones y amenazas; 3.4\% (9) reportaron castigo con labores pesadas, no se consignó en ninguno de los casos el suministro de drogas o medicamentos que el niño no necesitaba.

En relación al tipo de contusiones simples, el $29 \%$ (48) de los casos presentó equimosis, el $10.1 \%$ (17) excoriaciones y el $11 \%$ (18) presentó equimosis más excoriaciones. Respecto al tipo de lesiones reportadas, la distribución se muestra en el Cuadro 4, donde observamos que el $61.3 \%$ (164) de los casos presentó contusiones simples, y en el $32 \%$ (85) de los casos no se encontró ningún tipo de lesión.

En relación a las regiones anatómicas, el 32.3\% (86) presentó una región lesionada, siendo los miembros inferiores la región más frecuente; $y$ el 36.1\% (96) presentó múltiples regiones lesionadas, siendo más frecuente la presencia de lesiones conjuntas en la espalda, glúteos, miembros superiores e inferiores. 
Cuadro 1. Tipos de Maltrato Infantil

\begin{tabular}{lcc}
\hline Tipo de Maltrato & No de casos & Porcentaje\% \\
\hline Omisión & 20 & 7.5 \\
Supresión & 2 & 0.7 \\
Transgresión & $\mathbf{1 1 2}$ & $\mathbf{4 1 . 9}$ \\
Supresión y omisión & 6 & 2.2 \\
Transgresión y omisión & 12 & 4.5 \\
Transgresión y Supresión & 76 & 28.5 \\
Transgresión, Supresión y Omisión & 18 & 6.7 \\
Ninguno & 21 & 7.9 \\
Total & $\mathbf{2 6 7}$ & $\mathbf{9 9 . 9}$ \\
\hline
\end{tabular}

Fuente: Departamento de Clínica Forense de Tegucigalpa, Dirección de Medicina Forense

Cuadro 2. Maltrato infantil por omisión

\begin{tabular}{lcc}
\hline Maltrato por Omisión & № de casos & Porcentaje \% \\
\hline Ha sido abandonado & 15 & 27 \\
No va a la escuela & 14 & 25 \\
Permanece sin el cuidado de un adulto & 11 & 19.6 \\
Alimentación insuficiente e inadecuada & 7 & 12.5 \\
Inadecuada higiene corporal & 3 & 5.3 \\
Vivienda inadecuada & 3 & 5.3 \\
Permanece sin el cuidado de un adulto, higiene & & 5.3 \\
corporal inadecuada, vivienda inadecuada & 3 & $\mathbf{1 0 0 . 0}$ \\
Total & $\mathbf{5 6}$ & \\
\hline
\end{tabular}

Fuente: Departamento de Clínica Forense de Tegucigalpa, Dirección de Medicina Forense

Cuadro 3. Maltrato infantil por supresión

\begin{tabular}{lccc}
\hline Maltrato por Supresión & № de casos & Porcentaje \% \\
\hline Medidas correctivas & & \\
inapropiadas & 76 & 74.5 \\
No recibe atención médica cuando lo requiere & 13 & 12.7 \\
Expulsión del hogar & 10 & 9.8 \\
Restricción a la libre deambulación & 3 & 2.94 \\
Total & $\mathbf{1 0 2}$ & $\mathbf{1 0 0}$ \\
\hline
\end{tabular}

Fuente: Departamento de Clínica Forense de Tegucigalpa, Dirección de Medicina Forense 
Cuadro 4. Maltrato infantil, según tipo de lesiones

\begin{tabular}{lcc}
\hline Tipo de lesiones encontradas & № de casos & Porcentaje\% \\
\hline Contusiones complejas & 1 & 0.4 \\
Contusiones simples & $\mathbf{1 6 4}$ & $\mathbf{6 1 . 3}$ \\
Fractura de cráneo, contusión cerebral leve & 1 & 0.4 \\
Fractura de dedo índice derecho & 1 & 0.4 \\
Intento de asfixia mecánica & 4 & 1.5 \\
Quemaduras por contacto & 7 & 2.7 \\
Quemaduras por escaldadura & 3 & 1.1 \\
Quemaduras por flama & 1 & 0.4 \\
Ninguna & 85 & 32.0 \\
Total & $\mathbf{2 6 7}$ & $\mathbf{1 0 0 . 0}$ \\
\hline
\end{tabular}

Fuente: Departamento de Clínica Forense de Tegucigalpa, Dirección de Medicina Forense

En dos casos la víctima negó el maltrato físico, encontrando al examen físico hallazgos de contusiones simples; en 50 casos que afirmaban haber sufrido maltrato físico no se evidenció lesiones. En relación a la compatibilidad de las lesiones por maltrato infantil en el $76.7 \%$ (205) de los casos se dio compatibilidad con objeto o cuerpo romo. Se realizaron estudios radiológicos en el $42.3 \%$ (113) de los casos, reportándose lesiones únicamente en dos casos.

El $93.4 \%$ (250) no presentó secuelas o limitación de la actividad física. En el 99.2\% (265) no hubo peligro de muerte, reportándose peligro de muerte solo en dos casos, el primero, un menor de dos años con quemaduras por escaldadura en cabeza, cara, cuello y miembros superiores y el otro, un menor de un año con maltrato por omisión y supresión por recibir una alimentación insuficiente e inadecuada y no recibir atención médica cuando lo requirió.

Se recomendó evaluación por Trabajo Social Forense en el 94.8\% (253) de los casos, encontrando que solo un caso fue evaluado. El
$1.5 \%$ (4) de los casos fueron evaluados por Odontología forense.

En la relación agresor-víctima observamos que el $47.2 \%$ (126) de los casos corresponde a la madre y el $24 \%$ (64) al padre. La presencia de relaciones conflictivas de pareja se observó en $24 \%$ (64) de los agresores.

\section{DISCUSIÓN}

En general se sabe relativamente poco sobre la prevalencia de algunas formas de maltrato, particularmente fuera del contexto de América del Norte ${ }^{5}$ y se estima que las tasas difieren en razón del diseño del estudio, la metodología de recolección de la muestra y de la definición de maltrato utilizada. Landsfor y colaboradores indican que casi una cuarta parte de los adultos $(22,6 \%)$ ha sufrido abuso físico en la infancia; más de un tercio (36.3\%) han sufrido abuso emocional infantil y el $16,3 \%$ ha sufrido negligencia infantil ${ }^{6}$.

En nuestro país aún no hay estudios de prevalencia de maltrato infantil, sin embargo, en este estudio encontramos que el maltrato infantil por transgresión, que se refiere a las acciones o 
conductas hostiles o destructivas, malos tratos físicos, suministrar drogas o medicamentos innecesarios, la sobrealimentación, las agresiones emocionales como la ofensa y la humillación o el castigo por medio de labores pesadas ${ }^{7-9}$; se reportó en el $41.9 \%$ de los casos, siendo el maltrato físico el que más se encontró (95.5\%). Varios autores han reportado que el castigo físico se sigue usando de manera habitual en algunas familias y países; y se estima que su uso se relaciona a factores asociados a la cultura y creencias de los progenitores y cuidadores, así como a la estrategia disciplinaria con que los padres fueron disciplinados cuando eran niños ${ }^{10,11}$. UNICEF en su informe del 2017 reportó que uno de cada cuatro cuidadores, afirmaron que el castigo físico es necesario para criar o educar adecuadamente a los niños y que tres de cuatro niños son habitualmente víctimas de algún tipo de disciplina violenta por parte de sus cuidadores $^{12}$.

Respecto al maltrato por omisión, que se refiere a cuando el niño es dejado solo, a la imposibilidad al acceso de necesidades básicas, intelectuales, recreativas y emocionales ${ }^{7-9}$, se observó en el 7.5\% de los evaluados; encontrándose que el $27 \%$ de los niños habían sido abandonados, el $25 \%$ no iba a la escuela y el $19,6 \%$ permanecía sin el cuidado de un adulto. En general no se conoce ampliamente la magnitud de este tipo de maltrato; Tran y colaboradores reportaron que hay menos estudios de negligencia que para cualquier otra categoría de maltrato y que los estudios sobre el abandono físico en los países de "bajos recursos" están notoriamente ausentes ${ }^{13}$.

El maltrato por supresión se refiere a tratos discriminatorios, la aplicación de medidas disciplinarias o correctivas que generan daño físico o moral a través de medidas coercitivas, la expulsión del hogar, la restricción de la libre deambulación, el derecho a la atención médica y los medicamentos que requiere ${ }^{7-9}$; se observó en el $0.7 \%$ de los evaluados, correspondiendo el $74,5 \%$ a medidas correctivas o disciplinarias inadecuadas para la edad.

El $28.5 \%$ de los casos se presentaron asociados al maltrato por transgresión y supresión, y el 13,4\% presentaron varios tipos de maltrato asociado, lo que concuerda con lo afirmado por Milla: "Es raro encontrar un niño en el que el maltrato sea de un solo tipo, un niño golpeado es también maltratado emocionalmente; un niño que evidencia signos de falta de cuidado, frecuentemente padece maltratos físicos o emocionales" ${ }^{\prime 7}$. En el $7.9 \%$ no fue posible demostrar maltrato.

Nuestros resultados indican que las niñas son más maltratadas que los varones $(57,3 \%, t=2,408$, $\mathrm{df}=266$, pvalor=0,016) si se analizan conjuntamente todas las formas de maltrato, , lo que concuerda con lo reportado en un estudio previo realizado en nuestro país ${ }^{14}$; por otra parte Tran y colaboradores estimaron que la probabilidad de abuso infantil era de 12 a $17 \%$ para las niñas y de 5 a $8 \%$ para los niños ${ }^{13}$, Mwakanyamale et al en $2018^{15}$, encontraron en un estudio realizado en Tanzania, que las mujeres presentaban una prevalencia más alta de abuso físico (84.3\%), negligencia (28.0\%) y abuso sexual (26.2\%) en relación a sus contrapartes masculinas que mostraron abuso emocional más frecuentemente (53.3\%). Moody y colaboradores concluyeron que las tasas de prevalencia media de abuso físico en Norteamérica, fueron de $24.3 \%$ y $21.7 \%$ similares para niños y niñas respectivamente, al igual que las tasas reflejadas en Asia que fueron similares para ambos sexos; en los estudios europeos, el abuso físico fue mucho mayor en los niños $(27,0 \%)$ que en las niñas $(12,0 \%)$. Asimismo, encontraron que las tasas de 
prevalencia de abuso emocional entre las niñas $(28,4 \%)$ en América del Norte fueron el doble que en los niños $(13,7 \%)$, en Europa fueron $6,2 \%$ en niños y $12,9 \%$ en niñas y en Asia, fueron más altas para los niños $(33,2 \%)$ que para las niñas $(26,9 \%)^{5}$. Respecto a la omisión reportan que las tasas de prevalencia fueron mucho más altas para las niñas de América del Norte $(40,5 \%)$ que para los niños (16,6\%); en Asia fueron similares para los niños $(23,8 \%)$ y las niñas $(26,3 \%)$, lo que también sucedió en Europa, y encontró un número muy pequeño de estudios en los continentes restantes (África, Australia y estudios de América del Sur). Por otro lado, Stoltenborgh reportó que la prevalencia global estimada fue de 163 / 1,000 para la negligencia física y de 184 / 1,000 para la negligencia emocional, sin diferencias aparentes de género ${ }^{16}$.

Los niños evaluados procedían con mayor frecuencia del área urbana, lo que podría ser explicado en términos de accesibilidad a los entes de persecución penal. El 72\% fueron agredidos en su vivienda. El $46 \%$ convivían con su familia nuclear, el $25.8 \%$ con su familia extendida y el $13,5 \%$ con familia desintegradas, contrastando con lo reportado en un estudio previo realizado en un hospital público de Honduras entre 1978 a 1983 que reportó que el $44.1 \%$ de los casos convivía en familias desintegradas y el $41 \%$ con familias integradas ${ }^{10}$.

El tiempo promedio transcurrido entre el hecho y la evaluación médico legal fue de 19 días, solo en el $50 \%$ de los casos la evaluación pericial fue menor a tres días, lo que refleja el incumplimiento de la notificación obligatoria de $\mathbf{2 4}$ horas siguientes al hecho, que exige la ley hondureña ${ }^{2,17}$, varios autores señalan que en general los médicos asistenciales no identifican, ni denuncian sistemáticamente los casos de abuso infantil, siendo algunas de las limitantes para la denuncia la falta de capacitación orientada no solo a la identificación del maltrato, sino también en la gestión, en la adecuada interacción con los órganos legales correspondientes y al seguimiento en la gestión y resultados ${ }^{1814}$, se mencionan además las relacionadas a la certeza en saber cómo identificar todas las formas de maltrato, incluidas las menos comunes y menos evidentes como el maltrato emocional, la negligencia física o emocional, el abuso contra niños con discapacidades, etc. Otro factor referido es el contexto laboral del notificador que incluye la sobrecarga laboral que genera el proceso, el poco apoyo institucional, el impacto en la familia como estigma por la divulgación del maltrato, el bajo nivel de confianza en los servicios de protección al menor o instituciones responsables, los efectos negativos sobre el notificador o incluso sobre el mismo niño posterior a la denuncia etc. ${ }^{18,19}$, los resultados de esta metasíntesis realizada sobre las experiencias del personal responsable de realizar la notificación obligatoria de los casos concluye que es necesario evaluar la efectividad de la notificación obligatoria, ya que no se ha encontrado evidencia de calidad que demuestre la efectividad de esta medida ${ }^{19}$.

Respecto al tipo de lesiones encontramos que las contusiones simples son las más frecuentes con el 61.3\% de los casos. La región anatómica más lesionada fueron los miembros inferiores en el $32.3 \%$ y en $36.1 \%$ de los casos lesiones conjuntas en la espalda, glúteos, miembros superiores e inferiores, en el $76.7 \%$ de los casos; las lesiones fueron compatibles con objeto o cuerpo romo, siendo el instrumento más frecuente el cinturón, hallazgos similares a los encontrados en el Instituto de Medicina Legal de Colombia que reportó contusiones en el $70.5 \%$ de los $\operatorname{casos}^{20}$

Podemos concluir que los niños evaluados por maltrato infantil en la Clínica Forense de Tegucigalpa indican, son en su mayoría, niñas 
( $t=2,408, d f=266$, pvalor=0,016), en edad escolar, con 8.6 años de edad en promedio, procedentes del área urbana, que conviven en el seno de su familia nuclear, y que son agredidas en su vivienda. El maltrato físico fue el más frecuentemente observado, siendo el cinturón el principal objeto de agresión.

\section{REFERENCIAS BIBLIOGRÁFICAS}

1.-Schilling $S$, Christian CW. Child physical abuse and neglect. Child Adolesc Psychiatr Clin N Am. 2014;23 (2):309-19. doi: $10.1016 /$ j.chc.2014.01.001

2.- Honduras. Congreso Nacional. Código de la Niñez y la Adolescencia. Decreto No.35-2013. Reformas Integrales en Familia y Niñez. La Gaceta [Internet]. 6 septiembre 2013 [Consultado 8 noviembre 2018. Disponible en: http://www.rnp.hn/wpcontent/uploads/2014/01/Decreto-352013Reformas-Codigo-de-la-Ninez-yAdolescencia.pdf

3.-Butchart A, Phinney Harvey A. Prevención del maltrato infantil: qué hacer, y cómo obtener evidencias. [Internet]. Ginebra; OMS; 2009 [Consultado 29 agosto 2016]. Disponible en:

http://apps.who.int/iris/bitstream/10665/442 $\underline{\text { 28/1/9789243594361 spa.pdf }}$

4.-R Core Team. R: A Language and Environment for Statistical Computing. [Internet].Vienna; R Foundation for Statistical Computing; 1999-2012.[Consultado el 10 de Agosto de 2018]. Disponible en: https://cran.rproject.org/doc/manuals/r-

release/fullrefman.pdf

5.- Moody G, Cannings-John R, Hood K, Kemp A, Robling $M$. Establishing the international prevalence of self-reported child maltreatment: a systematic review by maltreatment type and gender. BMC Public Health [Internet]. 2018.[Consultado el 10 de agosto de 2018].;18(1):1164. Disponible en: https://www.ncbi.nlm.nih.gov/pubmed/30305 $\underline{071}$

6.- Lansford JE, Alampay LP, Al-Hassan S, Bacchini D, Bombi AS, Bornstein $\mathrm{MH}$, et al. Corporal punishment of children in nine countries as a function of child gender and parent gender. Int J Pediatr. 2010;2010:672780. doi:10.1155/2010/672780.

7.-Milla V. Maltrato Infantil. Hondur Pediátr [Internet]. 2003 [Consultado el 12 de mayo de 2018];23(2):167-72. Disponible en http://cidbimena.desastres.hn/RHP/pdf/2003/ pdf/Vol23-2-2003-10.pdf

8.- Rodríguez Ochoa LE. Abordaje del maltrato infantil su conceptualización la valoración médica y protocolos de atención. [Tesis Internet]. Ecuador: Universidad Técnica de Machala; 2017. [Consultado el 14 de agosto de 2018]. Disponible en: https://bit.ly/2S2hX49

9.-Rodríguez Ramírez MP, Serón Díaz T, Rostion C. Maltrato infantil. Rev Ped Elec [Internet]. 2014 [Consultado 16 de julio 2016];11(4):1622. Disponible en: https://bit.ly/2B9UxAj

10.-Sauceda-García JM, Olivo-Gutiérrez NA, Gutiérrez J, Maldonado-Durán JM. El castigo físico en la crianza de los hijos. Un estudio comparativo. Bol Med Hosp Infant Mex [Internet]. 2006 [Consultado 10 enero 2018];63(6):382-88. Disponible en: http://www.scielo.org. mx/pdf/bmim/v63n6/v 63n6a4.pdf

11.-Watakakosol $R$, Suttiwan $P$, Wongcharee $H$, Kish A, Newcombe PA. Parent discipline in Thailand: corporal punishment use and associations with myths and psychological 
outcomes. Child Abuse Negl. 2019; 88:298306. DOI: 10.1016/i.chiabu.2018.12.002. [Epub ahead of print]

12.-Fondo de las Naciones Unidas para la Infancia. Una situación habitual: violencia en las vidas de niños y los adolescentes. [Internet]. NY: UNICEF; 2017[Consultado 16 de julio 2018]. Disponible en: https://www.unicef.org/publications/files/Viol ence in the lives of children Key findings $S$ p.pdf

13.-Tran BX, Pham TV, Ha HG, Ngo AT, Nguyen $\mathrm{LH}, \mathrm{Vu}$ TTM, et al. A bibliometric analysis of the global research trend in child maltreatment. Int J Environ Res Public Health. 2018;15(7): E1456.

14.-Tejeda Suazo IM. Síndrome del niño maltratado, revisión de egresos en el Hospital Materno Infantil, período de 1978 a 1983. [Tesis Internet]. Tegucigalpa: UNAH; 1984 [Consultado 14 julio 2016]. Disponible en: http://www.bvs.hn/TMH/pdf/TMH25/pdf/TM H25.pdf

15.-Mwakanyamale AA, Wande DP, Yizhen Y. Multi-type child maltreatment: prevalence and its relationship with self-esteem among secondary school students in Tanzania. BMC Psychol. 2018;6(1):35. DOI: 10.1186/s40359018-0244-1.
16.-Stoltenborgh $M$, Bakermans-Kranenburg MJ, Van IJzendoorn MH. The neglect of child neglect: a meta-analytic review of the prevalence of neglect. Soc Psychiatry Psychiatric Epidemiol. 2013;48(3):345-55.

17.- Honduras. Congreso Nacional. Código de la niñez y la adolescencia. Decreto No. 73-96. La Gaceta [Internet]. 5 septiembre 1996 [Consultado 29 agosto 2016]. Disponible en: https://www.unicef.org/honduras/codigo nin $\underline{\text { ez adolescencia.pdf }}$

18.-Flaherty EG, Sege R. Barriers to physician identification and reporting of child abuse. Pediatr Ann. 2005;34(5):349-56.

19.-McTavish JR, Kimber $M$, Devries $K$, Colombini M, MacGregor JCD, Wathen CN, et al. Mandated reporters' experiences with reporting child maltreatment: a meta-synthesis of qualitative studies. BMJ Open.2017;7(10):e013942. DOI:10.1136/bmjopen-2016-013942

20.- Valenzuela Cortés D. Maltrato infantil en niñas 2004-2008. [Internet]. Colombia: Centro de Referencia Nacional sobre Violencia; 2010 . [Consultado 14 agosto de 2015]. Disponible en: http://www.medicinalegal.gov.co/documents/ 20143/56311/MALTRATO.pdf 